\title{
Política y mecenazgo anticuario en la Roma del Renacimiento: el caso de Bernardino López de Carvajal
}

\author{
Politics and antiquarian patronage in Renaissance \\ Rome: the case of Bernardino López de Carvajal
}

\author{
Paloma MARTÍN-ESPERANZA \\ Facultad de Filosofía y Letras. Universidad Autónoma de Madrid \\ https://orcid.org/0000-0002-1438-9542 \\ paloma.martin-esperanza@uam.es
}

\begin{abstract}
Cardinal Carvajal gathered at his residence in Rome an archaeological collection as a sign of the prestige and power he had achieved in his political-ecclesiastical career, but also his profile as a humanist and patron. Through two antiquarian sources of the time, the so-called Note d'anticaglie (1499-1501) and the codices of Pietro Sabino (c. 1490-1495), a reconstruction of Carvajal's archaeological collection is proposed, including the identification of the inscriptions by the $\mathrm{CIL}$ number, and some hypotheses are raised about their participation in the excavations of Villa Adriana. These sources have also led to the discovery of a Roman sarcophagus belonging to an orator Hispaniarum; this confirms not only the tendency among the elites of the period towards the incipient collecting, but also the importance of antiquities in the self-representation of cardinals and ambassadors.
\end{abstract}

Keywords: Collecting; Quattrocento; Antiques; Bernardino López de Carvajal; Cardinals.
Resumen: El cardenal Carvajal reunió en su residencia de Roma una colección arqueológica como signo del prestigio y poder que había alcanzado en su faceta político-eclesiástica, pero también en su perfil de humanista y mecenas. A través de dos fuentes anticuarias de la época, la llamada Nota d'anticaglie (1499-1501) y los códices de Pietro Sabino (c. 1490-1495), se propone una reconstrucción de la colección arqueológica de Carvajal, incluyendo la identificación de las inscripciones mediante el número de CIL, y se plantean algunas hipótesis sobre su posible participación en las excavaciones de Villa Adriana. Igualmente, se aborda la discusión en torno a la presencia en las fuentes anticuarias de un sarcófago romano perteneciente a un orator Hispaniarum, lo que confirma no solo la tendencia entre las elites hacia el incipiente coleccionismo, sino también la importancia de las antigüedades como medio de autorrepresentación para los cardenales y embajadores españoles afincados en Roma.

Palabras clave: Coleccionismo; Quattrocento; Antigüedades; Bernardino López de Carvajal; Cardenales.

* Este trabajo ha sido posible gracias a dos estancias de investigación en Roma, la primera en la Università degli Studi di Roma «Tor Vergata» (2018), financiada por el Servicio de Investigación de la Universidad Autónoma de Madrid y dirigida por la Prof. Beatrice Cacciotti, y la segunda en la Sapienza Università di Roma (2019), bajo la dirección de la Prof. Flavia Cantatore, a quienes agradezco todas sus aportaciones a este estudio. 
A finales del siglo XV, Bernardino López de Carvajal y Sande (Cáceres, 1456Roma, 1523), cardenal de Santa Croce in Gerusalemme y embajador de los Reyes Católicos ante la Santa Sede, reunió en su residencia de Roma una colección arqueológica, formada por unas pocas inscripciones y una estatua marmórea, como signo del prestigio y poder que había alcanzado en su faceta político-eclesiástica, pero también en su perfil de humanista y mecenas. Inmerso en el vasto panorama de relaciones sociales y culturales que se vivía en la Roma del tránsito del Quattrocento al Cinquecento, el cardenal Carvajal, fruto del creciente prestigio de Fernando e Isabel en la ciudad papal, gozó de una amplia influencia sobre los principales artistas e intelectuales de su época, sobre los que ejerció un mecenazgo activo donde la Antigüedad, en general, y las antigüedades, en particular, tuvieron un papel determinante ${ }^{1}$. Así se extrae de los códices anticuarios y epigráficos que se incluyen en este trabajo y que constituyen una fuente de sumo interés para el estudio de la figura de Bernardino de Carvajal, a pesar del nulo tratamiento que han suscitado en la historiografía sobre el cardenal.

No cabe duda de que el coleccionismo se constituyó, a finales del Quattrocento, como un fenómeno al que paulatinamente se adscribirían los cardenales de Roma, «les principaux exportateurs de marbres antiques» ${ }^{2}$, debido al marcado carácter mecenático de la Curia y a la personalidad cosmopolita desarrollada como fruto de su actividad diplomática -nunciaturas y legaciones-. Lo cierto es que, en las últimas décadas del siglo XV, la acumulación de objetos en los palacios y residencias de Roma comenzó a teñirse de una connotación de prestigio que demostraba no sólo el poder económico del coleccionista, sino también su

1 Sobre Bernardino López de Carvajal resultan imprescindibles los trabajos de Flavia CANTATORE, Un committente spagnolo nella Roma di Alessandro VI: Bernardino Carvajal, en VV.AA., Roma di fronte all'Europa al tempo di Alessandro VI, Atti del Convegno, Città del Vaticano-Roma, 1-4 de diciembre de 1999, 3 vols. Roma, 2001, III, pp. 861-871 y Don Bernardino López de Carvajal: Cardenal, Embajador y casi Papa, en José Manuel MARTín GaLÁN, Arte y diplomacia en el reinado de los Reyes Católicos, 2002, pp. 127-132; Isabella IANNUZZI, Bernardino de Carvajal: teoria e propaganda di uno spagnolo all'interno della Curia romana, en Rivista di storia della Chiesa in Italia, 62/1 (2008), pp. 25-45 y Le radici culturali di uno spagnolo alla corte papale: Bernardino de Carvajal, en Metafore di un pontificato. Giulio II (1503-1513) (Roma, 2-3 de diciembre de 2008), Roma, 2010, pp. 45-59; Álvaro FERNÁNDEZ DE CóRdova, López de Carvajal, Bernardino, en Diccionario Biográfico Español, vol. XXX, 2009, pp. 395-401; además de Marco BONA CASTELlOTTI, A proposito di Bernardino Carvajal comittente, en Arte lombarda, 51, 1979 y José GOÑ GAZTAMBIDE, Bernardino López de Carvajal y las bulas alejandrinas, en Anuario de Historia de la Iglesia, 1 (1992), pp. 93-112.

2 Patricia Falguières GuIdiCELLI, La cité fictive. Les collections de cardinaux, à Rome, au XVI siècle, en André CHastel (ed.), Les Carrache et les Décors Profanes (Roma, 2-4 de octubre 1986), École Française de Rome, Rome, 1988, p. 223. 
conocimiento de los antiguos ${ }^{3}$. Si bien en un primer momento el coleccionismo cuatrocentista estuvo limitado, en su mayoría, a la recopilación de manuscritos y objetos litúrgicos, así como a gemas y otros objetos 'pequeños' -véanse las colecciones del cardenal Orsini ${ }^{4}$, de Paulo II $^{5}$, del cardenal Gonzaga ${ }^{6}$ o del cardenal Bessarione ${ }^{7}$, entre otros-, a partir de la segunda mitad del siglo XV observamos una paulatina preferencia por las estatuas y los epígrafes, como prueban las colecciones del cardenal Prospero Colonna ${ }^{8}$ o de Giuliano della Rovere, cuya exhibición, en sus residencias privadas o en sus propias iglesias, contribuía a una ostentación de su status social y cultural. A este aspecto habría que sumar el alto valor económico que tenían estas piezas, lo que hacía del coleccionismo una oportunidad comercial nada despreciable, a la que se sumarán también las familias de la alta burguesía romana ${ }^{10}$. De este modo, las colecciones de Pietro y Mario Mellini ${ }^{11}$, Francesco Porcari ${ }^{12}$, Andrea Santacroce ${ }^{13}$ o Paolo della Valle ${ }^{14}$ dominarán el panorama coleccionista del último tercio del siglo XV.

3 Ibid., p. 249.

4 Luigi Gaetano MARINI, Degli archiatri pontifici, I, Roma, 1784, pp. 131-132; Eugène MüNTZ, Les arts à la cour des Papes pendant le XV $V^{e}$ et le XVI ${ }^{e}$ siècles, III: Sixte IV - Léon X (1471-1521), Ernest Leroux, París, 1882, p. 177.

5 Eugène MüNTZ, op. cit.; Anna CAVAllaro (ed.), Collezioni di Antichità a Roma tra '400 e '500, De Luca Editori d'Arte, Roma, 2007, p. 10.

6 Eugène MÜNTZ, op. cit, p. 297-300; David S. CHAMBERS, A Renaissance cardinal and his worldly goods: the Will and inventory of Francesco Gonzaga (1444-1483), London, 1992, pp. 132-137. El inventario de bienes del cardenal Francesco de Gonzaga, guardado en el Archivio di Stato de Mantua, ha sido publicado por Chambers, cfr. Anna CaVallaro, Collezioni... [ver n. 6], p. 10.

7 Anna CaVallaro, Antoniazzo romano e gli antoniazzeschi, Udine, 1992, pp. 54-60.

8 Sara Magister, Censimento dell collezioni di antichità a Roma: 1471-1503, en Xenia Antiqua, VIII (1999), p. 160.

9 Para la colección de Giuliano della Rovere véase Sara MAgISTER, Le collezione di Antichità del cardinale Giuliano della Rovere. Anteprima di studio, en Xenia Antiqua, IX (2000), pp. 47-50.

10 Anna Cavallaro, Collezioni... [ver n. 6], p. 11.

11 Sara Magister, Censimento... [ver n. 9], p. 174; Sandro SANTOLIni, Pietro e Mario Millini fondatori di una dinastía di collezionisti antiquari, en Anna CAVALLARO (ed.), Collezioni di Antichità a Roma tra '400 e '500, De Luca Editori d'Arte, Roma, 2007, pp. 39-62.

12 Sara Magister, Censimento... [ver n. 9], p.182; Mara MinASI, Passione politica e travestimento all'antica: la collezione antiquaria della familia Porcari, en Anna CAVAlLARO (ed.), Collezioni di Antichità a Roma tra '400 e '500, De Luca Editori d'Arte, Roma, 2007, pp. 83-104.

13 Sara MAgISTER, Censimento... [ver n. 9], p.185; Francesca VICARELLI, La collezione di antichità della familia Santacroce, en Anna CAVAlLaRo (ed.), Collezioni di Antichità a Roma tra '400 e '500, De Luca Editori d'Arte, Roma, 2007, pp. 63-82.

14 Sara Magister, Censimento... [ver n. 9], p.164; Maria Cristina PAOluZZI, La familia della Valle e l'origine della collezione di antichità, en Anna CAVAlLARO (ed.), Collezioni di Antichità a Roma tra '400 e '500, De Luca Editori d'Arte, Roma, 2007, pp. 147-186. 
A esta época corresponden también las colecciones arqueológicas de los cardenales españoles residentes en la Roma cuatrocentista ${ }^{15}$, destacando la de Rodrigo de Borja (1431-1503) ${ }^{16}$, quien reunió en su magnífico palacio de Roma, la «Cancelleria vecchia», actual palacio Sforza-Cesarini (construido entre 1457 y 1462 en la vía Banco di S. Spirito esquina con el Corso Vittorio Emanuele II), una interesante colección epigráfica, dispuesta en el jardín ( $\ll$ nella sua corte per terra» ${ }^{17}$; «in stabulis» ${ }^{18}$; «in quodam pariete post stabulum $»^{19}$ ), que sin duda contribuyó a la magnificencia del palacio que nos transmiten sus contemporáneos ${ }^{20}$. A ésta cabe sumar, ahora, la colección arqueológica de Bernardino López de Carvajal, nutrida, como en el caso anterior, fundamentalmente de epígrafes, lo que no es del todo común si tenemos en cuenta que, todavía en el Quattrocento, el coleccionismo era fundamentalmente ecléctico y volcado hacia lo arqueológico, especialmente hacia los objetos de pequeño tamaño ${ }^{21}$ o, en el caso de la escultura, el coleccionismo quedaba limitado al ámbito de disfrute personal, del gusto ${ }^{22}$. Respecto al origen de las piezas, como ha señalado Cavallaro, la modalidad de adquisición a finales del

$15 \mathrm{El}$ amplio panorama de las relaciones diplomático-culturales de los españoles en Roma ha sido magistralmente recogido por Álvaro FERNÁNDEZ DE CÓRDOVA, Imagen de los Reyes Católicos en la Roma pontificia, en En la España medieval, 28 (2005), pp. 259-354. A este respecto, véase también Ángel GómeZ Moreno, España y la Italia de los humanistas: primeros ecos, Madrid, 1994; Carlos José HERNANDO SÁNCHEZ (coord.), Roma y España. Un crisol de la cultura europea en la Edad Moderna, 2 vols., Madrid, 2007, y Devid PAOLINI, Los Reyes Católicos e Italia: los humanistas italianos y su relación con España, en Nicasio SALVADOR MigueL y Cristina MOYA GARCÍA (eds.), La literatura en la época de los Reyes Católicos, Pamplona-Madrid-Frankfurt, 2008, pp. 189-205.

16 Paloma Martín-EsPeranza, Alejandro VI y las antigüedades de Roma: coleccionismo, excavaciones y spolia, en Revista Borja. Revista de l'IIEB, 6 (2017-2018), pp. 1-28.

17 ms. Florencia, Biblioteca Marucelliana, A. 78. 1, f. 62, cf. Sara Magister, Censimento... [ver n. 9].

18 ms. Roma, Biblioteca Apostólica Vaticana, Vat. lat. 10228, f. 54v y ms. Roma, BiblioteCa APOSTÓlica VATICANa, Borg. lat. 336, f. $28 v$.

19 ms. Verona, Biblioteca Capitular, 270, f. 28v; ms. Venecia, Biblioteca Marciana, lat. XIV 171, 4665, f. 45v; ms. VENECIA, MUSEO CORRER, 1632, f. 66; ms. FlorenCIA, BiblioTeCa Nazionale Centrale, XXVIII. 34, f. 51., cf. Sara Magister, Censimento... [ver n. 9].

20 Guicciardini señala que el palacio estaba «pieno di mobili di grandissima valuta», Francesco Guicciardini, Storia d'Italia, VI/2, ed. por S. SEIDEL MENCHI, Turín, 1971, p. 459 y Enea Silvio Piccolomini, entonces papa Pio II, se atrevió a compararla con la Domus Aurea de Nerón, Enea Silvio Piccolomini, I Commentarii, ed. Giuseppe BernetTi, 5 vol., Cantagalli, Siena, 19721976, véase Paloma MARTín-EsPeranZA, Alejandro VI... [n. 17].

21 José BELTRÁN, Los procesos de amortización de los soportes epigráficos en la antigüedad y en época moderna, en Joan CARBOnELL, Helena GIMENO y José Luis MORALEJO (eds.), El monumento epigráfico en contextos secundarios. Procesos de reutilización, interpretación y falsificación, Universitat Autònoma de Barcelona, Barcelona, 2011, p.58.

22 El nombre de Bernardino de Carvajal ya aparece en el censo de Sara Magister, Censimento... [ver n. 9], p. 157. Gloria MORA, La escultura clásica y los estudios sobre la Antigüedad en España en 
siglo $\mathrm{XV}$ y principios del siglo XVI, especialmente para los epígrafes, pasaba por su encuentro fortuito en los terrenos de propiedad del coleccionista o por las excavaciones -sin método alguno- que se llevaban a cabo «nella stessa area di abitazione urbana» de Roma ${ }^{23}$. Tampoco podemos perder de vista que, con frecuencia, las piezas pasaban sistemáticamente de una colección a otra. Posiblemente, el origen de las colecciones de Borja y Carvajal hay que situarlo entre estas tres posibilidades, resultando de sumo interés sus permanentes relaciones con otros coleccionistas, a través de los cuales pudieran haber adquirido algunas de sus inscripciones.

A pesar de que la gran mayoría de las colecciones cuatrocentistas se encuentran perdidas o desmembradas, estando sus piezas repartidas entre los distintos museos europeos, es posible reconstruirlas gracias a los códices epigráficos y anticuarios de la época -muchos de ellos custodiados por la Biblioteca Apostólica Vaticana $^{24}$ - destacando, entre otros, las obras de Fray Giocondo (1497-1499) ${ }^{25}$, de Pietro Sabino (c. 1490-1495) ${ }^{26}$, de Giovanni Marcanova (1457-1460 y 1465) ${ }^{27}$, de Michel Fabrizio Ferrarino ${ }^{28}$ o de Battista di Pietro Brunelleschi (1509-1513) ${ }^{29}$ o la edición de Mazzocchi $(1521)^{30}$. Cabe incluir, también, fuentes literarias como

el siglo XVI: colecciones, tratados y libros de diseños, en Matteo MANCINI (coord.), El coleccionismo de escultura clásica en España: actas del simposio: 21 y 22 de mayo de 2001, Madrid, 2001, p. 118.

23 Anna Cavallaro, Collezioni... [ver n. 6], p. 16.

24 La mayoría de ellos pueden consultarse en formato digital y han sido bien estudiados por Marco BUONOCORE, Tra i codici epigrafici della Biblioteca Apostolica Vaticana, 2004, Faenza y Daniela Gionta, Epigrafia umanistica a Roma, 2005, Messina. También puede consultarse el catálogo de manuscritos de Bartolomeo Sanvito (1435-1518), en Albinia C. DE LA MARE y Laura NUVOLONI, Bartolomeo Sanvito: The Life and Work of a Renaissance Scribe, Anthony Hobson y Christopher de Hamel (eds.), London, 2009.

25 CIL, VI, p. XLIV, nr. XV. Véase Marco BUONOCORE, La raccolta epigrafica di Giocondo: metodo e manoscriti, en Pierre Gros y Pier Nicola PAGLIARA, Fra Giovanni Giocondo umanista, architetto e antiquario, Venezia, 2014, pp. 335-352. Sobre los códices epigráficos de la Biblioteca Apostólica Vaticana

26 CIL, VI, p. XLV, nr. XVI. Para la cronología véase Gerard GONZÁLEZ GERMAIN, La silloge epigrafica di Pietro Sabino: un riesame della tradizione manoscritta, en Epigraphica, 78 (2016), pp. 315-335.

27 CIL, VI, p. XLII, nr. VIII. Entre otros, Elisabetta BARILE, Per la biografia dell'umanista Giovanni Marcanova, Treviso, 2011.

28 CIL, VI, pp. XLIII-XLIV, nr. XIV. Véase Xavier EsPLUGA, Michele Fabrizio Ferrarini, en Epigraphica: periodico internazionale di epigrafia, 70 (2008), pp. 255-267.

29 CIL, VI, p. XLV, nr. XIX. Véase Heikki SoDIN, Appunti su Battista Brunelleschi epigrafista, en O. MERISAlO y R. VAINIO (eds.), Ad itum liberum. Essays in honour of Anne Helttula, Jyväskylä, 2007, pp. 79-92.

30 Nos referimos al ejemplar del Epigrammata Anriquae Urbis (1521), editado por Mazzocchi y anotado por Jean Matal (ca. 1517-1597), ms. Roma, Biblioteca ApOstóliCA VATiCANA, Vat. Lat. 8495. Sobre esta edición véase Joan CARBONELl MANIL y Gerard GONZÁlEZ GERMAIN, Jean Matal and his annotated copy of the Epigrammata Antiquae Vrbis (Vat. Lat. 8495): the use of manuscript sources, en Veleia, 29 (2012), pp. 149-168. 
la Nota d'anticaglie et spoglie et cose maravigliose et grande sono nella cipta de Roma da vederle volentieri (1499-1501) o el Prospettivo Milanese (1493-1496). La colección del cardenal Carvajal aparece, como veremos, en la Nota d'anticaglie ${ }^{31}$ y en los códices de Pietro Sabino ${ }^{32}$.

\section{UN CARDENAL HUMANISTA, PROTECTOR Y MECENAS EN ROMA}

De sólida formación humanística, Carvajal se graduó en Salamanca en Artes y Teología como bachiller en 1472 y doctor en 1478, ocupando ese mismo año la cátedra de Prima del que hubiera sido su maestro, Pedro Martínez de Osma, quien había sido juzgado y condenado por sus posiciones heréticas ${ }^{33}$. Como testimonian las actas de las reuniones de claustro, Carvajal tuvo la oportunidad de conocer en Salamanca a Antonio de Nebrija, adalid del nuevo espíritu cultural que se vivía en Castilla en tiempos de Isabel ${ }^{34}$ y creador de un discurso político que anclaba sus raíces en la Hispania romana. Estos contactos resultan de sumo interés si tenemos en cuenta que, Carvajal, una vez instalado en Roma, desplegará todo un discurso político tendente a unir lazos entre Italia y España, para lo que recurrirá a argumentos históricos extraídos del pasado clásico, como se desprende, de una manera magistral, de la oración de prestación de obediencia que, en nombre de Fernando e Isabel, pronunció ante el recién electo papa Alejandro VI $(1492)^{35}$.

Carvajal había llegado a Roma, como arcediano de Toro y cubicularius pontificio, en una fecha anterior a 1482, año en el que pronunció una homilía en la

31 Anónimo, Nota d'anticaglie et spoglie et cose maravigliose et grande sono nella cipta de Roma da vederle volentieri, editado por Agnese FANTOZZI, Roma, 1994. Corresponde al ms. ROMA, BIBLIOTECA Di ARCHEOlogia E STORIA DELL'ARTE, $51 \mathrm{~A}$.

32 Hemos consultado los dos códices que conserva la Biblioteca Apostólica Vaticana, ms. ROMA, Biblioteca Apostólica VATiCANa, Chigi. I. V. 168. y ms. Roma, Biblioteca Apostólica VATICANA, Ottob. lat. 2015, si bien existen tres manuscritos más custodiados en la Bibliothèque Inguimbertine (Carpentras), la Biblioteca degli Uffizi (Florencia) y la Biblioteca Nazionale Marciana (Venecia). Sobre los manuscritos de Sabino, [ver n. 27].

33 Isabella IANNUZZI, Bernardino de Carvajal... [ver n. 2], pp. 25 y ss.

34 Ibid. [ver n. 2], p. 28.

35 Me refiero a la Oratio super prestanda solenni obedientia Sanctissimo D.N. Alexandro Pape .VI. ex parte Christianissimorum dominor[um] Fernandi \& Helisabe Regis \& Regine Hispanic: habita Rome in consistorio publico, publicada en Roma en 1493, Inc. ROMA, BIBLIOTECA APOSTÓLICA VATICANA, Inc. IV, 566 (int. 8), ISTC ic00221000, que precisamente estoy estudiando y transcribiendo en mi Tesis doctoral, bajo la dirección de la Prof. Gloria Mora Rodríguez (UAM) y el Prof. Álvaro Fernández de Córdova Miralles (UNAV). 
capilla papal con motivo de la celebración de la festividad de Todos los Santos ${ }^{36}$. Dos años antes, en 1480, con ocasión de su nombramiento como rector de la Universidad de Salamanca, Carvajal entró en contacto con Pedro González de Mendoza, pues entre sus objetivos estaba el de convencer al cardenal de que construyera en esta ciudad el colegio mayor que pensaba erigir en Valladolid ${ }^{37}$. Aunque fracasó en sus objetivos, Carvajal consiguió cultivar una amistad con los Mendoza que le catapultaría a Roma, pues precisamente fue enviado a dicha ciudad para defender los intereses de la familia ${ }^{38}$. Más adelante, en 1486, será nombrado embajador por Fernando e Isabel, incorporándose a la misión diplomática del conde de Tendilla, Íñigo López de Mendoza, que tenía como objetivo defender los intereses de la Corona y conseguir la bula de Cruzada en el momento final de la Reconquista. A partir de este momento, Carvajal permanecerá en Roma con el fin expreso de desplegar una compleja acción diplomática en defensa de los intereses de los monarcas.

Es en este contexto, en la década de finales de los ochenta y principios de los noventa del siglo XV, ya instalado en Roma, cuando Carvajal se alzará como una figura de primer nivel político e intelectual. A ello contribuyó, por un lado, el círculo de amistades que cultivó en Roma, destacando la familia Mellini, en cuyo palacio residió entre 1496 y 1504, regresando, más tarde, en 1517, y quienes poseían una de las principales colecciones arqueológicas del momento ${ }^{39}$. No cabe desdeñar, tampoco, la cercanía de Carvajal a Rodrigo de Borja, quien, ya en su época de vicecanciller, se convirtió en un apasionado protector del humanismo romano, siendo también coleccionista de antigüedades y manuscritos, cercano a los miembros de la Academia Pomponiana y a figuras como Lorenzo Beheim, Luis Podocataro o Marco Antonio Sabellico ${ }^{40}$. Por último, en el ascenso de Carvajal, es importante mencionar las victorias políticas de los Reyes Católicos, que, sin duda, aumentaron el prestigio de los españoles y exigieron a los embajadores desplegar toda una cultura política al servicio de la causa de los monarcas. Para ello, Carvajal entró en contacto con artistas de la talla de Bramante o Peruzzi, con

36 Sermo in die omnium sanctorum in Capella dni nostri Sixti Pape quarti astante sanctitate sua \& sacro cetu Carinalum habitur per Reuerendum dnm Bernardinum Caruajal Arcium \& Theologie professorem Anno salutis Millesimoquadrigentessimooctauegesimosecundo in testinis bellis uniuersa pene Italia laborante, pronunciado en Roma en 1482, ms. ROMA, BiblioteCA ApOstólica VATICANA, Vat.lat.13679.

37 Álvaro FERNÁNDEZ de CóRdova, López de Carvajal... [ver n. 2].

38 Isabella IANNUZZI, Bernardino de Carvajal... [ver n. 2], p. 28.

39 Sara Magister, Censimento... [ver n. 9], p. 174; Sandro SAnTolini, Pietro e Mario... [ver n. 12], pp. 39-62.

40 Paloma Martín-EsPeranZa, Alejandro VI... [n. 17]. 
quienes contó para las obras que, desde 1488, se llevaron a cabo en el monasterio de San Pietro in Montorio ${ }^{41}$, sin olvidar tampoco la restauración de la iglesia de Santa Croce in Gerusalemme, en la que participó, entre otros, Antonio de Sangallo el Joven ${ }^{42}$, o las obras en la iglesia de Santiago de los Españoles. Este interés por el arte y la arquitectura posiblemente le llevó a adquirir, junto al también orator Juan Ruiz de Medina, un ejemplar de la editio princeps del De architectura de Vitrubio, que pasó a España a través de los embajadores en $1499^{43}$.

Además de los artistas, Carvajal se erigió como amigo y protector de preeminentes humanistas de la talla de Paolo Pompilio, quien le dedicó el Panegiricum Carmen ad Carvajalis (1490) ${ }^{44}$ o Alessandro Cortesi, que escribiría el De triumphata Bassa, Almeria, Granata (1492) ${ }^{45}$ dentro de la corriente literaria que ensalzaba las victorias de Isabel y Fernando contra el infiel, a la que se sumarán las obras de los hermanos Verardi ${ }^{46}$. A esta corriente de exaltación de los monarcas se adscribirán, también, los Commentaria super opera diversorum auctorum de antiquitatibus loquentiumde de Annio de Viterbo, publicados en Roma en 1498 bajo el amparo del cardenal Carvajal, que incluía un capítulo dedicado a la historia de España donde, a través de la falsificación histórica, se trataba de proporcionar a los Reyes Católicos una genealogía antiquísima que arrancaba en el propio Túbal, el nieto de Noé. Precisamente la relación de los embajadores y cardenales con Annio de Viterbo merece, en lo concerniente al uso de la Antigüedad, un tratamiento más profundo ${ }^{47}$.

El dominico Annio de Viterbo (1432-1502), que destacó en vida por su conocimiento de las lenguas orientales, la astrología, la alquimia, la teología y por

41 Flavia Cantatore, Il Tempietto di Bramante nel monasterio di San Pietro in Montorio, Edizioni Quasar, Roma, 2017.

42 Luipolt C. Frommel, Progetto e archeologia in due disegni di Antonio da Sangallo il Giovane per Santa Croce in Gerusalemme, en S. Danesi SQUARZINA (ed.), Roma, centro ideale della cultural dell'antico nei secoli XV e XVI. Da Martino V al sacco di Roma, 1417-1527, Milano, 1989, p. 382.

43 Fernando MARÍAS, Los clientes del Tempietto: historia, intenciones y significados, en Flavia Cantatore (ed.), Il Tempietto di Bramante nel monasterio di San Pietro in Montorio, Edizioni Quasar, Roma, 2017, p. 130.

44 Ms. Roma, Biblioteca Apostólica Vaticana, Vat. Lat. 2222.

45 Inc. Roma, Biblioteca Apostólica Vaticana, Stamp. Ross. 7868, istC ic00940400.

46 Vemos, por ejemplo, cómo en el prólogo de la obra de Marcelino Verardi, Elegiae et carmine, se agradece la supervisión de Carvajal y Medina, Fernando MARÍAS, Los clientes... [ver n. 44], p. 142.

47 La bibliografía sobre Annio de Viterbo es muy extensa, por lo que remito simplemente a algunos de los estudios más recientes: Anyhony GRAFTON, Invention of Traditions and Traditions of Invention in Renaissance Europe: The Strange Case of Annius of Viterbo, en Anthony GRAFTON y Ann BLAIR (eds.), The Transmission of Culture in Early Modern Europe, Philadephia, 1990, pp. 8-38; José Antonio CABallero, Beroso y Giovanni Nanni (Annius Viterbensis): modelos para el relato de los tiempos míticos en la historiografía española, en Revue des études anciennes 111/1 (2009), pp. 197-215. 
sus actividades arqueológicas, ascendió, durante el pontificado de Alejandro VI, al cargo de Magister Sacri Palatii -el teólogo que aconsejaba al Papa ${ }^{48}$-, trabajando, igualmente, al servicio del cardenal Carvajal, para el que escribió sus Commentaria o Antiquitates $^{49}$, que fueron publicados en Roma, cuya primera edición data de $1498^{50}$.

La cercanía de Annio con el papa Alejandro VI queda patente en las exploraciones arqueológicas que realizaron juntos a Orvieto y Viterbo, donde excavaron una tumba etrusca ${ }^{51}$. Este interés de Annio por lo arqueológico nos lleva a pensar en una posible participación del dominico en las excavaciones que se llevaron a cabo en el Odeón de Villa Adriana en tiempos de Alejandro $\mathrm{VI}^{52}$, de las que da cuenta Pirro Ligorio (1514-1583): «Hoggidì, dove fu il suo antico anphitheatro o teatro, è posta la rocca moderna secondo scrive papa Pio secondo che la edificò; et papa Alexandro Borgia, poscia, anche esso vi fabricò, et si vedeva nel fosso della rocca alcuni vestiggi del teatro $[. ..] \gg^{53}$. Fue en estos trabajos, a cargo del arquitecto Lorenzo da Pietrasanta (1496), donde aparecieron las famosas esculturas de las Musas $^{54}$, «le quali furono già tolta da questo teatro nel tempo di papa Alexandro Borgia $\gg^{55} \mathrm{y}$, posiblemente, el conjunto de catorce máscaras procedentes de Tívoli conservadas en los Museos Vaticanos ${ }^{56}$.

48 Giovanni Baffioni y Paola MatTIANGELI, Annio da Viterbo: documenti e ricerche: volume I, Consiglio nazionale delle ricerche, Roma, 1981, p. 266.

49 Los manuscritos originales de la obra, hoy perdidos, fueron adquiridos por el cardenal Carvajal, quien se los habría llevado a España, según José Antonio CABALLERo LÓPEZ, Annio de Viterbo y la Historiografía española del siglo XV, en Humanismo y tradición clásica en España y América: VI Reunión Científica sobre Humanistas Españoles, mayo, 2001, León y San Pedro de Dueñas, España, 2002, p. 111.

50 De esta obra existen 19 ediciones, datadas entre 1498 y 1602, que dan cuenta de la amplísima difusión que alcanzó el texto, José Antonio CABALlero LópeZ, Annio de Viterbo... [ver n. 49], p. 113.

51 Ximo COMPany, Alexandre VI $i$ Roma. Les empreses artístiques de Roderic de Borja a Itàlia, Tres i Quatre, Valencia, 2002 (Biblioteca Borja, 1), p. 177; Ian CAMPBELL, Rescue Archaeology in the Renaissance, en Ilaria BIGNAMINI (ed.), Archives \& Excavations. Essays on the history of archeological excavations in Rome and southern Italy from the Renaissance to the nineteenth century, Roma: British School of Rome, 2004, p. 13; Walter STEPHENS, When Pope Noab Ruled the Etruscans: Annius of Viterbo and his Forged Antiquities, en MLN (Mod Lang Notes) 119/1 (2004), pp. 201-223; Paloma MARTÍN-ESPERANZA, Alejandro VI... [n. 17], p. 15.

52 Paloma Martín-EsPeranZa, Alejandro VI... [n. 17], p. 16.

53 Pirro LigOrio, Libro dell'antica città di Tivoli e di alcune famose ville, ed. Alessandra TEN, Roma, 2005 , p. 48.

54 Xavier Dupré I Raventós, Spagnoli a Villa Adriana, en Anna Maria REgGIANI (dir.), Villa Adriana. Paesaggio Antico e ambiente Moderno: elementi di novità e ricerche incorso (Atti del Convegno, Roma, Palazzo Massimo alle Terme, 23-24 giugno 2000), Electa, Roma, 2003, p. 125.

55 Pirro Ligorio, Libro dell'antica... [ver n. 54], pp. 48.69.

56 Véase el catálogo de los Museos Vaticanos <https://catalogo.museivaticani.va/opere/\#>. MartínEsperanza, 2019: 17. 
Por su parte, aunque no tenemos constancia directa de la participación de Carvajal en estas obras, debemos recordar su presencia en los alrededores de la Villa Adriana durante estos años, como prueba el hecho de que su nombre aparezca vinculado a las labores de adaptación del palacio de los gobernadores de Tívoli, precedente de la famosa villa del cardenal Ippolito d'Este, quien promocionó, a través de Pirro Ligorio, las excavaciones en la Villa ${ }^{57}$. Sabemos también que Bramante, por entonces al servicio de Carvajal, visitó la Villa en 1501, coincidiendo posiblemente con la fecha de las excavaciones de Alejandro $\mathrm{VI}^{58}$. Además, Fernando Marías ha señalado que hasta 1521 Carvajal fue gobernador en Tívoli ${ }^{59}$, lo que confirmaría aún más la presencia del cardenal de la Santa Croce en dichas incursiones arqueológicas.

Por último, la pericia arqueológica de Carvajal quedó patente en el hallazgo de las reliquias de la Cruz, concretamente del titulus crucis, que, tal y como nos transmite Bessozzi ${ }^{60}$, fue encontrado el mismo día que llegaron a Roma las noticias de la toma de Granada (1492) en las excavaciones que, amparadas por Bernardino López de Carvajal, se llevaban a cabo en la cripta de la basílica de Santa Croce in Gerusalemme ${ }^{61}$, coincidente con el cubiculum de la emperatriz Elena en la domus Sessoriana. El descubrimiento tuvo un fuerte eco en Castilla y, como ha señalado Felipe Pereda, «a modo de primicia de la arqueología cristiana», el

57 Treccani, Bernardino LÓPEZ DE CARVAJAL: <http://www.treccani.it/enciclopedia/bernardino-lopez-de-carvajal_\%28Dizionario-Biografico\%29/>.

58 Jack FreIBERG, Bramante's Tempietto, Roman Renaissance, and the Spanish Crown, Cambridge University Press, Cambridge, 2014, p. 78.

59 Fernando MARÍAS, Los clientes... [ver n. 44]. No hemos podido, sin embargo, confirmar este dato que aporta Marías, pues el nombre de Carvajal no aparece en la lista de los gobernadores de Tívoli recogida por Michele GIUSTINIANI, Due libri de vescovi e de governatori di Tivoli, Roma, 1665 .

$60 \ll$ La soffitta pure era dipinta, e divisa in quadri ornati nel mezzo con cose di rilievo dorate, eccetuatane quella parte in cui erano dipinte le arme del Re di Spagna e del Cardenale Mendozza, che ne fu l'autore. In mezzo v'erano le arme di marmo del Cardinale suddetto, e sopra di esse si vedeva una croce di due traversi dorata, la quale aveva un coperchio di marmo sostenuto da due sotili colonne, ed era collocata in un campo colorito di azzurro, in cui a caratteri d'oro si leggevano queste parole: Hic fuit Titulus Sanctae Crucis, che fu a caso ritrovato, come si dirà in appresso nell'ultima domenica di Gennaro dell'anno 1492 ; giorno in cui arrivó in Roma la notizia, che il Re Ferdinando aveva espugnata Granata per l'avanti da' Mori tiranicamente occupata», Raimondo BesozZI, La Storia Della Basilica Di Santa Croce in Gerusalemme, 1750, p. 30 .

61 Véase Felipe Pereda, Pedro González de Mendoza, de Toledo a Roma. El patronazgo de Santa Croce in Gerusalemme: entre la arqueología y la filología, en Frédérique LEMERLE, Yves PAUWELS, Gennaro TOSCANO (eds.), Les Cardinaux de la Renaissance et la modernité artistique, Publications de l'Institut de recherches historiques du Septentrion, Villeneuve d'Ascq, 2012, pp. 217-243. 
titulus fue inmediatamente copiado en el crucifijo de la Cartuja de Miraflores $(1496-99)^{62}$. No cabe duda de que Carvajal supo, en este caso, utilizar la fuerza del supuesto hallazgo arqueológico para enfatizar su mensaje político, que pasaba, irremediablemente, por situar a Isabel y Fernando como los continuadores de la obra de Elena y Constantino, esto es, como los restauradores del Imperio romano cristiano.

\section{CARVAJAL EN LAS FUENTES ANTICUARIAS: SU COLECCIÓN ARQUEOLÓGICA}

Visto el amplio panorama de relaciones artísticas e intelectuales de Carvajal, así como su interés por la Antigüedad y sus vestigia, parece lógico pensar que el cardenal se sumara a la incipiente moda del coleccionismo de antigüedades. En efecto, gracias a la Nota d'Anticaglie et spoglie et cose maravigliose et grande sono nella cipta de Roma da vederle volentieri ${ }^{63}$, manuscrito anónimo conservado en la Biblioteca di Archeologia e Storia dell'Arte (B.I.A.S.A.), fechado entre 1499-1501, sabemos que el cardenal Bernardino López de Carvajal (1456-1523) albergó en su casa una estatua de un joven, posiblemente antigua y en estado fragmentario $-\ll e t$ a chasa el cardinale di Santa Croce é un torso de giovane fuori fatto simile al vero» ${ }^{64}-$, a la que se sumarían, según nos transmiten varias antologías epigráficas $^{65}$, algunas inscripciones.

Esta pequeña colección arqueológica habría sido atribuida, erróneamente, por la editora de la Nota d'Anticaglie (1994), a la familia Santacroce, cuyo apellido coincide con el cargo que ostentó, a partir de 1495, Bernardino de Carvajal: el de cardenal de Santa Croce in Gerusalemme y, por tanto, referido en las fuentes bajo el epíteto de «cardinale di Santa Croce ${ }^{66}$. Cabría pensar, como han apunta-

62 Felipe Pereda, Pedro González de Mendoza... [ver n. 62], p. 15.

63 ANÓNIMO, Nota d'anticaglie... [ver n. 32], p. 17.

64 ANÓNIMO, Nota d'anticaglie... [ver n. 32], p. 17.

65 ms. Roma, Biblioteca Apostólica Vaticana, Chigi. I. V. 168; ms. Roma, Biblioteca ApoSTÓLICA VATICANA, Ottob. lat. 2015.

66 Igualmente, su predecesor en el título aparece así mencionado: «cardinalis Sanctae Crucis qui est Mendoza» (Stefano INFesSura, Diario de la città di Roma di Stefano Infessura scribasenato, ed. O. Tommasini, Roma, 1890, p. 270), aunque no así en Conradus EubEL, Hierarchia Catholica medii et recentioris aevi, sive summorum pontificum, S.R.E. cardinalium, ecclesiarum antistitum series volumen tertium saeculum ХVI ab anno 1503 complectens, Monasterii (Münster), 1923, pp. 22, 62 y 72, donde le vemos con los epítetos de Seguntino, Hispalensis o Toletanus. 
do algunos autores ${ }^{67}$, que la escultura podría haber pertenecido a algún cardenal de la noble familia Santacroce, sin embargo, como recoge el Hierarchia Catholica medii et recentioris aevi, sive summorum pontificum, S.R.E. cardinalium, ecclesiarum antistitum series volumen tertium saeculum XVI ab anno 1503 complectens, esta familia no tuvo ningún cardenal hasta $1569^{68}$.

Así, la confusión entre la colección del cardenal de Santa Croce con la de la familia Santacroce ${ }^{69}$, que desde el Quattrocento en adelante lograron reunir una importante variedad de materiales arqueológicos, es uno de los motivos por el cual la historiografía concerniente al cardenal Carvajal no ha prestado, hasta hoy, atención a su faceta coleccionista ${ }^{70}$.

Merece la pena rastrear el quid de esta equivocación historiográfica. La colección arqueológica de la familia Santacroce, que habría arrancado al inicio del Quattrocento con Andrea Santacroce, alcanzará su momento decisivo con Valerio Santacroce, quien, en la primera mitad del Cinquecento, expondrá sus mármoles en el cortile de su propio palacio ${ }^{71}$. Entre ambos la historiografía ha situado a Prospero Santacroce ${ }^{72}$, al que erróneamente atribuye Francesca Vicarelli la colección citada en la Nota d'Anticaglie ${ }^{73}$, ya que éste nunca fue cardenal y, por tanto, no puede hacer referencia al «cardinale de Santa Croce» citado en la misma. En un error similar caen Pray Rober y Rubinstein al atribuir a Prospero Santacroce el título de cardenal, confundiéndole con el futuro cardenal Prospero del Cinquecento (1513-1589) y al que, por las fechas, tampoco puede referirse

67 Phyllis Pray BOBER y Ruth RUBINSTEIN, Renaissance artists and antique sculpture, a Handbook of Sources, with contributions by Susan Woodford. Oxford University Press, Oxford, 1986, p. 177.

68 Conradus EuBeL, Hierarchia Catholica... [ver n. 67].

69 Cabe recordar que la familia Santacroce no tuvo ningún cardenal hasta el año 1565 , tal y como aparece en Conradus EubEL, Hierarchia Catholica... [ver n. 67].

70 Esta identificación errónea no aparece en Sara MAGISTER, Censimento... [ver n. 9], pp. 157 y 198.

71 La colección arqueológica de la familia Santacroce ha sido estudiada en profundidad por Kathleen Wren CHRISTIAN, From ancestral cults to art: the Santacroce collection of antiquities, en Walter CUPPERI (ed.), Senso delle rovine e riuso dell'antico (Annali della Scuola Normale Superiore di Pisa, IV, 14), Pisa, 2002, pp. 255-271; y Francesca VICARELLI, La collezione di antichità della familia Santacroce, en Anna CaVallaro (ed.), Collezioni di Antichità a Roma tra '400 e '500, De Luca Editori d'Arte, Roma, 2007, pp. 63-82.

72 Prospero Santacroce, laico, se dedicó a la actividad financiera y comercial hasta que, en 1480, Sixto IV ordenó que le fuera confiscado su patrimonio al ser acusado de homicidio en la confrontación con Pietro Margani, miembro de la nobleza «di campanile»-o nobleza menor- romana. Véase Roberto LANCIANI, Storia degli scavi di Roma e notizie intorno le collezioni romani di antichità, I (1000-1530), Roma, 1989, p. 157.

73 Francesca VICARELLI, La collezione... [ver n. 72], pp. 68-74. 
la Nota d'Anticaglie, compuesta entre el año 1499 y el $1501^{74}$. El hecho de que sí haya existido un cardenal de la familia Santacroce, aunque bastante más tardío, ha llevado a la confusión a los investigadores.

Tal y como ha estudiado Vicarelli, basándose en fuentes como el opúsculo Antiquarie prospettiche romane ${ }^{75}$ o el Prospectivo Melanese depictore ${ }^{76}$, además de las tradicionales antologías epigráficas del Quattrocento, como las de Fray Giocondo $^{77}$ o Pietro Sabino ${ }^{78}$, la colección de Prospero Santacroce estaría formada por varias esculturas antiguas, entre ellas una Venus Púdica ${ }^{79}$, una estatua de $\operatorname{Pan}^{80}$ y una amazona ${ }^{81}$. No hay rastro, en estas descripciones, del «torso de giovane» del que nos habla la Nota d'Anticaglie. Además, al atribuir las inscripciones del «cardenal Santa Croce» a Prospero Santacroce y no a Bernardino de Carvajal, la autora no repara en que, en el manuscrito Chigi. I. IV. 168, f. $62 \mathrm{r}$ aparecen las inscripciones de «Prosperi Ste. Crucis», mientras que en Chigi. I. $I V .168, f .62 v$, esto es, en el anverso del mismo folio, aparecen las del «cardinalis $s+»$, diferenciadas las unas de las otras, por lo que, en ningún caso, puede tratarse de la misma colección.

74 Phyllis Pray Bober y Ruth RUBInSTEIN, Renaissance artists... [ver n. 68], p.177.

75 Roma, Biblioteca Casanatense, Inc. 1628, editado por Doris D. Fienga, The Antiquarie Prospetiche Romane composte per Prospectivo Melanese Depictore: A document for the study of the relationship between Bramante and Leonardo Da Vinci, Tesis Doctoral (1970), University of California, 1971, Los Ángeles.

76 Gilberto GOVI, Intorno a un opusculo rarissimo della fine del secolo XV intitolato «Antiquarie Prospettiche Romane» composte per Prospettivo Milanese Dipintore, en Atti della Reale Accademia dei Lincei, $2^{\mathrm{a}}$ s., 3, 1876, pp. 49-66.; Doris D. FIENGA, The Antiquarie... [ver n. 76], con traducción al inglés.

77 ms. Roma, Biblioteca Apostólica VATiCANA, Vat. lat. 10228, f. 54v; ms. Roma, BiblioteCa Apostólica Vaticana, Borg. lat. 336, f. 28v; ms. Roma, BiblioteCa ApostóliCa Vaticana, Vat. Lat. 5326.

78 ms. Roma, Biblioteca Apostólica Vaticana, Borg. lat. 336, f. 28v; ms. Roma, Biblioteca Apostólica Vaticana, Chigi. I. V. 168.

79 Un dibujo de un artista anónimo, conservado en una colección privada, datado en el 1503, presenta una escultura de Venus junto a la que puede leerse, en letra manuscrita, «Misier Prospero», Cfr. Francesca VICARELLI, La collezione... [ver n. 72], p. 70, colección L. Bertini, fol. 9v, en Anne SCHмIтT, Römische Antikensammlungen im Spiegel eines Musterbuch der Renaissance, en Müncher Fabrbuch der bildenden Kunst, 21 (1970), pp. 104; 116, fig. 11. Además, Schmitt ha identificado este dibujo con otro contemporáneo, también de autor anónimo, del cuaderno de Holkham Hall, donde aparece una Venus con el siguiente apunte: «questa fémina sta in chasa/questa di santa/ chrocie e [una] bella chosa e tonda», en Holkham Hall, Ms. 701, f. 34vd.

80 Tal y como lo describe Prospectivo Melanese «Et ecci in casa dun di santa croce un nudo e tiene un zappo scorticato che dessere assai bono ha molta voce», Gilberto GOVI, Intorno... [ver n. 76]; Doris D. FIENGA, The Antiquarie... [ver n. 76], p.33, s. 35.

81 Anne SCHMITT, Römische... [ver n. 80], p. 125; Kathleen Wren CHRISTIAN, From ancestral... [ver n. 68], fig. 14b; VICARELLI, La collezione... [ver n. 72], pp. 73 y 74. 
Teniendo en cuenta estos datos, parece claro que el coleccionista cuatrocentista del que habla la Nota d'Anticaglie no puede ser otro que Bernardino de Carvajal, el auténtico cardenal de la Santa Cruz.

La faceta coleccionista de Bernardino de Carvajal encaja con su personalidad y sus relaciones, ya que, como han expresado los autores ${ }^{82}-\mathrm{y}$ desarrollaremos más adelante en este trabajo-, el cardenal ejerció una gran influencia en el ambiente artístico de la época ${ }^{83}$. Su interés por el arte y la arquitectura, y en particular por la Antigüedad, debió consolidarse, en parte, gracias a su paso por el «palatium commune familia de Milinis» ${ }^{84}$, en el barrio de Parione, concretamente, en la via S. Maria dell'Anima (via di Tor Millina), donde vivió entre 1496 y 1504 y, más tarde, en $1517^{85}$, y donde se conservaba una rica colección de antigüedades ${ }^{86}$ a la que hace referencia, entre otros, Prospectivo Melanese en su Antiquarie: «a dir duno misser Mario Mellino / irestarei sensa calamaro / se ben tenessi piu cuno amplio tino» ${ }^{87}$. En dicho palacio había residido anteriormente el también embajador Juan Ruiz de Medina ${ }^{88}$.

Como he señalado anteriormente, Pietro y Mario Mellini, miembros de una importante familia noble romana, consiguieron reunir una de las colecciones de antigüedades más importantes del Quattrocento ${ }^{89}$. De ella se hizo eco Polizia-

82 Isabella IANnUZzi, Bernardino de Carvajal... [ver n. 2]; Marco Bona CASTELlotTI, A propósito... [ver n. 2].

83 En palabras de Flavia Cantatore, «uno dei membri piú influenti della comunità iberica residente a Roma», Flavia CANTATORE, San Pietro in Montorio. La Chiesa dei Re Cattolici a Roma. Università degli Studi di Roma «La Sapienza». Ed. Quasar, Roma, 2007, p. 46. Sobre las relaciones de Carvajal con los artistas del Quattrocento, como Bramante o Bramantino, etc., véase Marco BoNA CASTEllotti, A proposito... [ver n. 2], p. 28.

84 Siguiendo las indicaciones de Paolo CORTESI, Libri de cardinalatu ad Iulium Secundum pontificem máximum, en Castro Cortesio, 1510, cap. II, de domo cardinalium, f. 50r; Archivio Serlupi Crescenzi, Famiglia Millini, vol. v, cc. 139 r-146 v (1523), cfr. Flavia CANTATORE, San Pietro in... [ver n. 83], p. 47; Véase también el Archivio Serlupi Crescenzi, Famiglia Millini, v, pergamene, n. 102, 2 giugno 1504, cfr. Simona SPERINDEI, Repertorio delle residenze cardenalizie, en Giorgio SIMONCINI (ed.), Roma. Le trasformazioni urbane nel Quattrocento. II. Funzione urbane e tipologie edilizie (L'Ambiente Storico. Studi di Storia urbana e del territorio, XI), Firenze, 2004, p. 143.

85 Simona SPERINDEI, Repertorio... [ver n. 85], p. 143; Micaela ANTONUCCI, Bramante nella Roma di Alessandro VI, tra mecenatismo spagnolo e comittenza «all'antica», en Ximo COMPANY, Borja FRANCO e Iván REGA (eds.), Bramante en Roma, Roma en España. Un juego de espejos en la temprana Edad Moderna, Universitat de Lleida, 2014, p. 84.

86 Sandro Santolini, Pietro e Mario Millini... [ver n. 12], pp. 39-62.

87 Gilberto GOvI, Intorno... [ver n. 76], p. 14; Doris D. FIENGA, The Antiquarie... [ver n. 72], p.43, s. 32.

88 Thomas J. DANDELET, La Roma española (1500-1700), Barcelona, 2002, p. 162.

89 Sandro SANTOLInI, Pietro e Mario Millini... [ver n. 12]. 
no en sus Miscellanea, donde considera el palacio un lugar repleto de mármoles romanos ${ }^{90}$. Tampoco podemos olvidar las palabras de Fray Mariano de Firenze, quien, en su Itinerarium Urbis Romae, afirmaba que en el barrio de Parione, habitaban escritores, auditores, oficiales, ciudadanos y prelados «in quorum domibus statuae pulcherrimae iliis», citando a los Mellini ${ }^{91}$. La colección tuvo como punto de partida el oratorio de Santa Croce, en la villa familiar de Monte Mario, donde, como explica Santolini:

In Santa Croce i Millini cominciano a raccogliere numerose epigrafi cimiteriali cristiane, incise su lastre di chiusura dei loculi sotterranei, testimonianza della proiezione della loro fede religiosa in un settore specifico del collezionismo $\operatorname{archeologico}^{92}$.

Además de la relación de amistad que habían establecido Mario Mellini y Bernardino de Carvajal, ambos compartían no sólo los mismos intereses culturales -como evidencia su actividad coleccionista- sino también la devoción por la Santa Cruz, leitmotiv de la vida de Carvajal, como prueban su título cardenalicio y sus esfuerzos por reformar la basílica de Gerusalemme in Santa Croce. Del mismo modo, Pietro y Mario Mellini terminaron de construir el oratorio de Santa Cruz de Monte Mario en 1470, levantado sobre la antigua capilla que Ponce de Péret, obispo de Orvieto y vicario del papa Clemente VI (1342-1352) había erigido en $1350^{93}$. Fue precisamente en aquel oratorio donde comenzaron a reunir la colección arqueológica que alcanzaría su plenitud en la casa de Parione.

Además de la estatuaria $-\ll$ statuae pulcherrimae iliis ${ }^{94}-$, conocemos la presencia de varias inscripciones romanas en la colección Mellini. Mazocchi registra

90 Miscellaneorum centuria prima, publicado en Florencia en 1498, Inc. ROMA, BIBLIOTECA APOSTÓliCa VatiCana, Stamp.Ross.1291, ISTC ip00890000. Véase William Parr GrEsWELl, Angelo Poliziano, Giovanni Pico della Mirandola, Jacopo Sannazaro, Pietro Bembo, Girolamo Fracastoro, Marco Antonio Flaminio, Girolamo Amalteo, Giovanni Battista Amalteo, and Cornelio Amalteo. Memoirs of Angelus Politianus, Joannes Picus of Mirandula, Actius Sincerus Sannazarius, Petrus Bembus, Hieronymus Fracastorius, Marcus Antonius Flaminius, and the Amalthei: Translations from Their Poetical Works: and Notes and Observations Concerning Other Literary Characters of the Fifteenth and Sixteenth Centuries, Cadell \& Davies, London, 1805, p. 91.

91 Cfr. Sandro Santolini, Pietro e Mario Millini... [ver n. 12], p. 47.

92 Ibid. [ver n. 12], p. 43.

93 «Sopra questo bel monte ornato di vigne e per la maggior parte posseduto dall'antica e nobile familia Mellini, fue da Pietro Mellini fondata questa picciol Chiesa [...] dedicandola alla santa croce l'anno 1470. A tre di maggio vi si celebra la festa», cfr. Sandro SANTOLINI, Pietro e Mario Millini... [ver n. 12], p. 42.

94 Cfr. Sandro Santolini, Pietro e Mario... [ver n. 12], p. 47; Fray Mariano da Firenze, Itinerarium Urbis Romae, ed. por BULlETTI O.F.M., Roma, 1931, p. 67. 
cuatro inscripciones funerarias «in domo d. Marii de Mellini» ${ }^{95}$ y otras dos «in domo domini Petripauli de Mellinis» ${ }^{96}$. Rodeado de este ambiente anticuario, Carvajal también se aventuró a engrandecer las colecciones del palatium Millinis, reuniendo para su residencia cardenalicia un conjunto de piezas romanas, como escribió en 1510 Francesci Albertini en su Opusculum de mirabilibus novae urbis Romae: «Domus Mellinorum est apud eccl. S. Mariae de Anima. Domus civum Rom. de Sca. Cruce cum pulcherr.statuis» ${ }^{97}$. Más tarde, según nos transmite el obispo de Scala, Baltasar del Río, Carvajal vivió los últimos años de su vida en el Palazzo Farnese ${ }^{98}$.

De la colección reunida por Carvajal durante su larga estancia en Roma cabe destacar, por un lado, las «pucherr.statuis» ${ }^{99}$, entre las cuales conocemos únicamente la existencia, gracias a la Nota d'Anticaglie, de un torso de un joven ${ }^{100} \mathrm{y}$, por otro, las inscripciones ${ }^{101}$. Son éstas últimas las que, quizás, más certezas nos aportan, ya que podemos aproximarnos a ellas gracias a los corpora antiguos.

La referencia a la domo cardinalis Sanctae + [Crucis] aparece en los códices de Pietro Sabino de la Biblioteca Apostólica Vaticana, Chig. I. V. 168, f. 62 v y Ottob. Lat. 2015, f. $21 \mathrm{v}$, que hemos consultado, en los que se recogen indistintamente dos inscripciones. A estas antologías se suma la Nota d'Anticaglie, que también hace referencia a la «chasa el cardinale Santa Croce». A partir del estudio de estas fuentes, hemos podido reconstruir la colección de Carvajal, que presentamos aquí, por primera vez, en forma de catálogo.

95 Cfr. Sandro Santolini, Pietro e Mario... [ver n. 12], p. 47; CIL, vI 9424, 16487, 20431, 27750.

96 Ibid. [ver n. 12], p. 48; CIL, VI 22811, 13434.

97 Francesco ALBERTINI, Opusculum de mirabilibus novae urbis Romae, ed. por A. SCHMARSOW, Heilbronn, 1886. El editor considera, en sus notas, que Albertini se refiere aquí a la residencia de la familia Santacroce, no obstante, parece improbable que el autor repitiera la misma idea, ya que, en la página anterior, señala: «Domus $S$. Crucis apud plateam Iudeor. sitae visuntur cum monumentis priscorum Romanorum», p. $29,88^{a}$, s. 25 , refiriéndose a la casa que los Santacroce tenían en la Piazza Giudea. Cabe tener en cuenta, además, que la referencia a la domus de Sca. Croce sigue a la de la domus Mellinorum, lugar de residencia de Carvajal.

98 Simona Sperindei, Repertorio... [ver n. 85], p.147; Marta Albalá Pelegrín, Humanism and Spanish Literary Patronage at the Roman Curia: The Role of the Cardinal of Santa Croce, Bernardino López de Carvajal (1456-1523), en Royal Studies Fournal (RS7), 4/2 (2017), p. 15.

99 Francesco ALBERTINI, Opusculum... [ver n. 97], p. 30, 88b, s. 20.

100 ANÓNIMO, Nota d'anticaglie... [ver n. 32], p. 17, rr. 41-42.

101 ms. Roma, BiblioteCa ApostóliCa VATiCANA, Chigi. I. V. 168; ms. Roma, BiblioteCa APOSTÓliCa VATICANA, Ottob. lat. 2015. 


\section{Catálogo}

Escultura

1) Nota d'Anticaglie

«un torso de giovane fuori fatto simile al vero»

Como ha señalado C.W. Christian, la descripción de un «torso de un joven» evoca la imagen del Pasquino, que habría sido trasladado desde el Palazzo Carafa a algún lugar indeterminado en las proximidades de la Piazza Navona, lo que nos lleva a pensar en que la famosa estatua estuviera en los alrededores del Palazzo Mellini ${ }^{102}$. No obstante, en el estado actual de la investigación, es difícil confirmar que la estatua del cardenal Carvajal se corresponda con el Pasquino.

\section{Inscripciones}

1) $C I L$ VI $2497=C I L$ VI $32651^{103}$

$\ll$ In domo Cardinalis s. + [Crucis]» (Pietro Sabino $)^{104}, \ll$ Propre S. Agnetem extra muros» (codex Cicognae Marucelliano) ${ }^{105}$, «In domo d. Marii de Melinis» (Fray Giocondo) ${ }^{106}$, «e regione dom. Millin.» (Pighius) ${ }^{107}$.

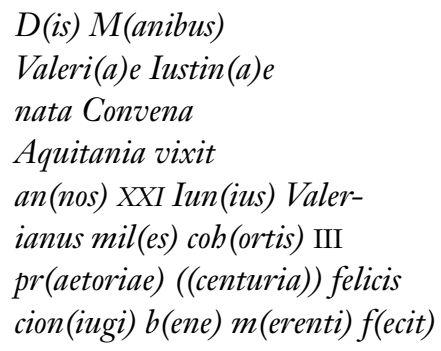

Ara marmórea procedente de Roma.

102 Kathleen Wren CHRISTIAN, Empire without end. Antiquities Collections in Renaissance Rome, 13501527, Yale University Press, New Haven, 2010, p. 346.

103 También aparece recogida en CIL X, *01089, 207, entre las inscripciones del Museo de Catania (Italia), esta vez como falso epigráfico. Habría llegado hasta Sicilia gracias a la labor de Placido Scammacca (1700 ca.-1787), responsable de la formación de la colección catanesi. Al respecto, véase Kalle Korhonen, Le iscrizioni del Museo Civico di Catania. Storia delle collezioni-Cultura epigrafica-Edizione (Commentationes Humanarum Litterarum, 121), Helsinki, 2004, p. 355. Giovannantonio DOSIO también incluye una edición de la inscripción en su libro Dis Manibus, pili, epitaffi et altre cose antiche, Pisa, 2011, p. 63.

104 ms. Roma, Biblioteca Apostólica VAticana, Chigi. I. V. 168; ms. Roma, Biblioteca APOSTÓlICA VATICANA, Ottob. lat. 2015.

105 ms. Florencia, Biblioteca Marucelliana, $A$ 79. 1 , f. 2.

106 ms. Florencia, Biblioteca Nazionale Centrale, Magl. XXVIII, 34, f. 43.

107 ms. Berlín, StaAtsbibliothek, Preubischer Kulturbesitz, Haus 2, Lat, f. 130. 
2) CIL VI 2243

In domo card. S. + [Crucis] ${ }^{108}$ (Pietro Sabino), «In palatio Mellinorum e regione S. Agnetis ad Agonem» (Marteen De Smet) ${ }^{109}$.

[H]ateria C[---]

sacer [dos ---]

ditis pa[tris ---]

Hateria

Helpis

Mater

Placa de mármol doctissima manu sculpta ${ }^{110}$ que incluye una representación de una mujer capite velata.

Los años en los que Carvajal reunió en el palatium Millinis su pequeña colección arqueológica, irremediablemente unida a la profusa colección de la noble familia, coincidieron con el mecenazgo artístico que, bajo su supervisión, llevaron a cabo los Reyes Católicos en Roma: la iglesia y el convento de San Pietro in Montorio, la iglesia y el hospital de Santiago de los Españoles y la iglesia de Santa Croce in Gerusalemme, todas ellas, empresas con un marcado carácter all'antico.

\section{EL SARCÓFAGO ROMANO DEL ORATOR HISPANORUM, ¿BERNARDINO LÓPEZ DE CARVAJAL, JUAN RUIZ DE MEDINA O GARCILASO DE LA VEGA?}

Son varios los códices epigráficos -Giocondo, Sabino, Anónimo de la Sovrintendenza- que dan noticia de un sarcófago antiguo, de mármol, custodiado «in quodam atrio ubi habitat orator Hispanorum»-«en el atrio donde habitaba el orador español»-, sin aportar más datos sobre su identidad ${ }^{111}$. La complejidad

108 ms. Roma, Biblioteca Apostólica Vaticana, Chigi. I. V. 168; ms. Roma, Biblioteca ApoSTÓliCa VATICANA, Ottob. lat. 2015.

109 ms. LEIDEN, BIBL. DER RIJKSUNIVERSITEIT, PBL, 1, ed. 46.

110 ms. LeIDEN, Bibl. Der RijKsuniversiteit, PBL, 1, ed. 46. También aparece en la síloge de Mazzocchi que conserva la Biblioteca Apostólica Vaticana, con las anotaciones realizadas por Jean Matal hacia 1550, quien la describe como «tabula marmórea», ms. ROMA, BIBLIOTECA ApOstólica VATICANa, Vat. Lat. 8495, f. $93 v$.

111 Así lo recoge Sara MagisTer, Censimento... [ver n. 9], pp. 177; Sara MagisTer, Censimento dell collezioni di antichità a Roma (1471-1503): addenda, en Xenia Antiqua, X (2001), p. 141. 
que entraña la identificación del personaje nos obliga a entrecruzar múltiples datos, comenzando por el propio epíteto de este, «orator Hispanorum», esto es, embajador de los españoles.

Durante el reinado de los Reyes Católicos, la diplomacia internacional adquirió un cariz humanista, tanto desde el punto de vista filológico como sociopolítico, motivado por el redescubrimiento del pensamiento clásico ${ }^{112}$. Como señala Nicasio Salvador, los embajadores «solían contar con una buena formación cultural, en la que entraba el dominio del latín» ${ }^{113}$, imprescindible si tenemos en cuenta que, más allá de la negociación técnica y letrada, la diplomacia de finales del siglo XV se caracterizó por sus orationes, esto es, por los discursos elaborados por los oratores, que tenían como principal objetivo ensalzar la personalidad de los príncipes o «explicar las motivaciones de una determinada alianza $\gg^{114}$. Es de recibo pensar, por tanto, que el personaje del que nos hablan los códices es un embajador. Entre los representantes de Fernando e Isabel no faltaron los agentes que utilizaron sus habilidades humanísticas para su servi$\operatorname{cio}^{115}$.

Ahora bien, se trata de un embajador «Hispanorum», no «regis Castellae» o «regis Aragonae». Esto nos lleva a pensar en un cuerpo diplomático unificado, que fue efectivo durante el pontificado de Inocencio VIII y especialmente durante el de Alejandro VI. A esta época corresponden nombres como Bernardino López de Carvajal (1487-1493), Juan Ruiz de Medina (1483-1485), Garcilaso de la Vega (1494-1499), Lorenzo Suárez de Figueroa (1499-1501) y Francisco de Rojas (1501-1507) ${ }^{116}$. Precisamente, la labor de estos diplomáticos coincide con los años de redacción de los códices epigráficos donde se menciona el sarcófago, todos ellos redactados entre 1488 y 1499. Para cerrar más el círculo, la cuarta redacción del códice de Giocondo (1504-1507) cita el sarcófago en los siguientes términos: «Romae in quodam atrio ubi habitabat orator Hispanorum, in arca

112 Álvaro FERNÁNDEZ De CÓRDOVA MIRALles, Diplomáticos y letrados en Roma al servicio de los Reyes Católicos: Francesco Vitale di Noya, Fuan Ruiz de Medina y Francisco de Rojas, en Dicenda: Estudios de lengua y literatura españolas, 32 (2014), p. 115.

113 Nicasio Salvador Miguel, Intelectuales españoles en Roma durante el gobierno de los Reyes Católicos, en Patrizia BOTTA (coord.), Rumbos del bumanismo en el umbral del cincuentenario de la A[sociación] I[nternacional de] H[ispanistas], Roma, 2012, p. 55.

114 Álvaro FERnáNDEZ De CóRdova MiRALLES, Diplomáticos... [ver n. 112], p. 115.

115 En el servicio diplomático de los Reyes Católicos encontramos humanistas como Antonio Geraldini, Joan Margarit, Antonio Agustín o Francesco Vitale de Noya, entre otros, cfr. Álvaro FERnández De Córdova Miralles, Diplomáticos... [ver n. 113], p. 115 y ss.

116 Nicasio Salvador Miguel, Intelectuales... [ver n. 114], p. 55. 
marmorea oblonga». El hecho de que se refiera al embajador en pasado, «babitabat», nos hace pensar que éste ya no se encontraba en Roma en las fechas en que fue redactado el códice. Quedarían descartados, en este sentido, Bernardino López de Carvajal y Francisco de Rojas, pues todavía estaban en Roma en estos años. Cabría pensar entonces en Juan Ruiz de Medina, Garcilaso de la Vega y Lorenzo Suárez de Figueroa.

Podríamos pensar que el orator Hispanorum del que hablan las fuentes es Juan Ruiz de Medina (c. 1440-1507), que permaneció en Roma hasta 1499, residiendo, precisamente, en Tor Millina. Obispo de Astorga (1489), Badajoz (1493) y de Cartagena (1495), Medina llegó a Roma en el séquito de Tendilla (1486), a cuyo éxito nos hemos referido anteriormente. Nombrado por los Reyes Católicos procurador ordinario en la Curia, junto con Bernardino López de Carvajal, aparece asociado a éste desde 1488, con quien compartió la responsabilidad de la construcción de San Pietro in Montorio en el Gianicolo ${ }^{117}$. Hombre de gran cultura, aparece en 1492 rodeado de humanistas de la talla de los hermanos Ve$\operatorname{rardi}^{118}$, cuyas composiciones para las celebraciones de la toma de Granada -Historia Baetica (1492), Fernandus Servatus (1493)- reflejan el espíritu de admiración por Isabel y Fernando que se vivía en la Roma de finales del Quattrocento. Son, precisamente, estos contactos con el ambiente intelectual de la época, así como su preeminencia y sus relaciones con Carvajal, las que llevan a pensar que el orator Hispanorum al que se refieren los códices es Juan Ruiz de Medina. No obstante, existe un detalle que podría alejarnos de esta certeza, y es que Medina era obispo $\mathrm{y}$, por tanto, parece lógico que este título apareciese en las fuentes, por encima del de orator ${ }^{119}$.

El siguiente nombre que podríamos barajar es el de Garcilaso de la Vega, orator hasta 1499 y laico. A diferencia de Medina, Garcilaso se relacionó más con el famoso Annio de Viterbo y tuvo intervenciones peculiares sobre la antigüedad del español, lo que manifiesta su sensibilidad por las cuestiones arqueológicas ${ }^{120}$. Sin embargo, en el estado actual de la investigación, no podemos llegar a una conclusión plausible.

\footnotetext{
117 Álvaro Fernández De Córdova Miralles, Diplomáticos... [ver n. 113], p. 124.

118 Ibid. [ver n. 113], p. 124.

119 Agradezco este dato al Prof. Álvaro Fernández de Córdova, que amablemente me advirtió de este detalle.

120 Como señala Freiberg, Carvajal y Garcilaso eran receptivos a las teorías de Annio dada su utilidad para la legitimación española, Jack FREIBERG, Bramante's... [ver n. 59], p. 117.
} 


\section{1) CIL VI 13447}

«In quodam atrio ubi habitat orator Hispanorum in arca marmorea oblonga ${ }^{121}$ (Sabino; Anónimo de la Sovrintendenza); «Romae in quodam atrio ubi habitar orator Hispanorum» ${ }^{122}$ (Giocondo); «Romae in quodam atrio ubi habitabat orator Hispanorum, in arca marmorea oblonga» (Giocondo) ${ }^{123}$.
$D($ is) M(anibus)
Baberia(e) Prima(e)
donavit sarco-
fagu $(m)$ donabit
singulari Aurelia
(H)armonia

Sarcófago de mármol, procedente de Roma.

\section{CONCLUSIÓN}

Gracias a los códices epigráficos y a las fuentes anticuarias recogidas en este estudio, es posible acercarse desde una óptica novedosa a la figura del cardenal Carvajal, situándole dentro del marco del coleccionismo de antigüedades de la Roma renacentista. Esta faceta de Carvajal aporta datos no solo sobre su personalidad, humanista y erudita, mecenas de artistas como Bramante, Peruzzi o Sangallo y frecuente de los círculos humanistas de Roma, sino que apoya también la premisa de que la posesión de antigüedades se concebía, incluso para los prelados españoles, como un instrumento legitimador y de prestigio, correspondiendo al nivel que se exigía de los embajadores de los Reyes Católicos. Así lo corroboran no sólo las pocas inscripciones y la escultura que albergó Carvajal en Tor Millina, sino también la colección de Rodrigo de Borja y el hecho de que un orator hispaniarum, todavía sin identificar, albergara en su residencia romana un sarcófago clásico. Este estudio ha permitido reunir, en forma de catálogo, las piezas que conformaban la colección de Carvajal, identificando las inscripciones y la estatua -quizás el famoso Pasquino de Roma- entre las posesiones del cardenal de Santa

121 ms. Roma, Biblioteca Apostólica Vaticana, Chigi. I. V. 168, f. 52; ms. Roma, BiblioteCa Apostólica Vaticana, Ottob. lat. 2015, f.2; ms. Venecia, Biblioteca NaZionale MarciaNA, Ms. lat. X 195 (3453); ms. FlORENCIA, BiblioteCA DELla SOVRINTENDENZA Di FirenZE, 7, II, f. 5 .

122 ms. VENECIA, MUSEO CORRER, 1632, f. 125.

123 ms. Florencia, Biblioteca LaurenZiana, Asbburnbamianus 905. (836), F. 95 (49). 
Croce, es decir, de Carvajal, y no del cardenal Próspero Santacroce ${ }^{124}$, como habían señalado erróneamente otros autores.

Además, se abre una nueva hipótesis al plantear la posible participación de Annio de Viterbo y de Carvajal en las excavaciones realizadas bajo el pontificado de Alejandro VI en Villa Adriana, atendiendo, principalmente, al hecho de que el cardenal de la Santa Croce aparezca vinculado a los trabajos de restauración del palacio de los gobernadores de Tívoli, sede de la futura Villa d'Este, en el momento en el que, según Pirro Ligorio, Alejandro vi habría llevado a cabo las excavaciones en el Odeón. Cabe señalar cómo el papa Borja se encargó de reforzar la figura de los magistri viarium para organizar y racionalizar las numerosas intervenciones arqueológicas, constructivas o restauradoras de los monumentos antiguos, por lo que su supervisión de los trabajos en la Villa está más que justificada. Queda abierta, no obstante, la cuestión de la participación de Carvajal en estos trabajos, hasta aclarar la función que tenía en el palacio de los gobernadores y si desempeñaba, o no, dicha dignidad en Tívoli.

En definitiva, el mecenazgo anticuario de Bernardino López de Carvajal permite situar al cardenal, una vez más, entre las figuras del panorama intelectual de Roma, como prueban no sólo sus relaciones eruditas, sino sus propios frutos literarios, refiriéndonos a sus orationes, que constituyen una síntesis de su pensamiento político, donde el recurso al argumento de la Antigüedad es constante. La faceta arqueológica de Carvajal, definida gracias a las fuentes anticuarias que aquí se presentan, constituye una nueva ventana desde la que acercarse a la interesante y polivalente figura de este embajador, teólogo, catedrático, humanista, mecenas, reformador y, en definitiva, cardenal de la Santa Cruz.

\section{REFERENCIAS BIBLIOGRÁFICAS}

\section{Referencias archivisticas}
a) Manuscritos

ms. Berlín, StaAtsbibliothek, Preubischer Kulturbesitz, Haus 2, Lat, f. 130. ms. Florencia, Biblioteca Marucelliana, $A$ 78. 1.

ms. Florencia, Biblioteca Marucelliana, $A 79$.

ms. Florencia, Biblioteca Nazionale Centrale, XXVIII. 34.

${ }^{124}$ Phyllis Pray Bober y Ruth RUBINSTEIN, Renaissance artists... [ver n. 67], p. 177. 
ms. Florencia, Biblioteca Della SovrintendenZa Di Firenze, 7, II. ms. Florencia, Biblioteca LaUREnZIANa, Asbburnhamianus 905. (836). ms. LeIDEN, BibliotheEk Der RijKSUNIVERSITEIT, PBL, 1, ed. 46. ms. ROMA, Biblioteca ApOSTÓlica VATICANA, BoRG. LAT. 336. ms. Roma, Biblioteca Apostólica VATICANA, CHIGI. I. V. 168. ms. ROMA, BiblioteCa ApOSTÓliCA VATICANA, OTTOB. LAT. 2015. ms. ROMA, BibLIOTECA APOSTÓliCA VATICANA, VAT. LAT. 10228. ms. ROMA, BiblioteCA ApOSTÓliCA VATICANA, VAT. LAT. 2222. ms. Roma, Biblioteca ApOSTÓlica VATICANA, VAT. LAT. 5326. ms. Roma, Biblioteca Apostólica VATiCANA, VAT. LAT. 8495. ms. Roma, BiblioteCa di ARCHEOlogia E STORIA DELL'ARTE, $51 \mathrm{~A}$. ms. Venecia, Biblioteca NaZionale Marciana, LaT. XIV 171, 4665. ms. Venecia, Biblioteca NaZionale Marciana, LAT. X 195 (3453) ms. VENECIA, MUSEO CORRER, 1632.

ms. VERona, Biblioteca CAPitular, 270.

\section{b) Incunables}

Inc. ROMA, BIBLIOTECA APOSTÓLICA VATICANA, Inc. $\mathrm{IV}, 566$ (int. 8). Inc. ROMA, BIBLIOTECA APOSTÓlICA VATICANA, Stamp. Ross. 7868. Inc. Roma, BIBLIOTECA APOSTÓliCA VATICANA, Stamp. Ross. 1291.

\section{Referencias bibliográficas}

Albalá Pelegrín, Marta, Humanism and Spanish Literary Patronage at the Roman Curia: The Role of the Cardinal of Santa Croce, Bernardino López de Carvajal (1456-1523), en Royal Studies Fournal (RS7), 4/2 (2017), pp. 11-37.

AlBERTINI, Francesco Opusculum de mirabilibus novae urbis Romae, ed. por A. SCHMARSOW, Heilbronn, 1886.

ANÓNIMO, Nota d'anticaglie et spoglie et cose maravigliose et grande sono nella cipta de Roma da vederle volentieri, B.I.A.S.A., Ms. 51 A, editado por Agnese FANTOZZI, Roma, 1994.

ANTONUCCI, Micaela, Bramante nella Roma di Alessandro VI, tra mecenatismo spagnolo e comittenza «all'antica», en Ximo COMPANY, Borja FrANCO e Iván REGA (eds.), Bramante en Roma, Roma en España. Un juego de espejos en la temprana Edad Moderna, Universitat de Lleida, 2014, pp. 79-91.

Baffioni, Giovanni y Mattiangeli, Paola, Annio da Viterbo: documenti e ricerche: volume I, Consiglio nazionale delle ricerche, Roma, 1981.

BARILE, Elisabetta, Per la biografia dell'umanista Giovanni Marcanova, 2011, Treviso.

BELTRÁn, José, Los procesos de amortización de los soportes epigráficos en la antigüedad y en época moderna, en Joan CARBOnELl, Helena GIMENO y José Luis MORAlEJO (eds.), El monu- 
mento epigráfico en contextos secundarios. Procesos de reutilización, interpretación y falsificación, Universitat Autònoma de Barcelona, Barcelona, 2011.

BesozzI, Raimondo, La Storia Della Basilica Di Santa Croce in Gerusalemme, 1750.

BOBER, Phyllis Pray y RUBINSTEIN, Ruth, Renaissance artists and antique sculpture, a Handbook of Sources, with contributions by Susan Woodford. Oxford University Press, Oxford, 1986.

Bona Castellotti Marco, A proposito di Bernardino Carvajal comittente, en Arte lombarda, 51 (1979), p. 28.

BuONOCORE Marco, Tra i codici epigrafici della Biblioteca Apostolica Vaticana, Faenza, 2004.

- La raccolta epigrafica di Giocondo: metodo e manoscriti, en Pierre GROS y Pier Nicola PAGLIARA (eds.), Fra Giovanni Giocondo umanista, architetto e antiquario, Marsilio, Venezia, 2014, pp. 335-352.

Caballero LópeZ, José Antonio, Annio de Viterbo y la Historiografía española del siglo XV, en Humanismo y tradición clásica en España y América: VI Reunión Científica sobre Humanistas Españoles, mayo, 2001, León y San Pedro de Dueñas, España, 2002, pp. 102-120.

- Beroso y Giovanni Nanni (Annius Viterbensis): modelos para el relato de los tiempos míticos en la bistoriografía española, en Revue des études anciennes, 111/1 (2009), pp. 197-215.

CAmpbell, Ian, Rescue Archaeology in the Renaissance, en Ilaria BIGNAMINI (ed.), Archives of Excavations. Essays on the history of archeological excavations in Rome and southern Italy from the Renaissance to the nineteenth century, British School of Rome, Roma, 2004, pp. 13-22.

CANTATORE, Flavia, Un committente spagnolo nella Roma di Alessandro VI: Bernardino Carvajal, en VV.AA, Roma di fronte all'Europa al tempo di Alessandro VI, Atti del Convegno, Città del Vaticano-Roma, 1-4 de diciembre de 1999, 3 vols. Roma, 2001, III, pp. 861-871.

- Don Bernardino López de Carvajal: Cardenal, Embajador y casi Papa, en José Manuel Martín García (ed.), Arte y diplomacia en el reinado de los Reyes Católicos, 2002, pp. 127-132.

- Il Tempietto di Bramante nel monasterio di San Pietro in Montorio, Quasar, Roma, 2017.

- San Pietro in Montorio. La Chiesa dei Re Cattolici a Roma. Università degli Studi di Roma «La Sapienza», Ed. Quasar, Roma, 2007.

CARbOnell MANIL, Joan y GONZÁlEZ GERMAIN, Gerard, Jean Matal and his annotated copy of the Epigrammata Antiquae Vrbis (Vat. Lat. 8495): the use of manuscript sources, en Veleia, 29 (2012), pp. 149-168.

Cavallaro, Anna (ed.), Collezioni di Antichità a Roma tra '400 e '500, De Luca Editori d'Arte, Roma, 2007.

- Antoniazzo romano e gli antoniazzeschi, Udine, 1992.

CHAMBERS, David S., A Renaissance cardinal and his worldly goods: the Will and inventory of Francesco Gonzaga (1444-1483), London, 1992.

Christian, Kathleen Wren, Empire without end. Antiquities Collections in Renaissance Rome, 1350-1527, Yale University Press, New Haven, 2010.

- From ancestral cults to art: the Santacroce collection of antiquities, en Walter CUPPERI (ed.), Senso delle rovine e riuso dell'antico (Annali della Scuola Normale Superiore di Pisa, IV, 14), Pisa, 2002, pp. 255-271.

COMPANY, Ximo, Alexandre VI $i$ Roma. Les empreses artístiques de Roderic de Borja a Itàlia, Tres i Quatre (Biblioteca Borja, 1), Valencia, 2002.

DANDELET, Thomas J., La Roma española (1500-1700), Barcelona, 2002. 


\section{POLÍTICA Y MECENAZGO ANTICUARIO EN LA ROMA DEL RENACIMIENTO}

De la Mare, Albinia C. y Nuvoloni, Laura, Bartolomeo Sanvito: The Life and Work of a Renaissance Scribe, Anthony Hobson y Christopher de Hamel (eds.), London, 2009.

Dosio, Giovannantonio, Dis Manibus, pili, epitaffi et altre cose antiche, Pisa, 2011.

Dupré I Raventós, Xavier, Spagnoli a Villa Adriana, en Anna Maria REgGiani (dir.), Villa Adriana. Paesaggio Antico e ambiente Moderno: elementi di novità e ricerche incorso (Atti del Convegno, Roma, Palazzo Massimo alle Terme, 23-24 giugno 2000), Electa, Roma, 2003, pp. 125-139.

ESPLUGA, Xavier, Michele Fabrizio Ferrarini, en Epigraphica: periodico internazionale di epigrafia, 70 (2008), pp. 255-267.

EubEL, Conradus, Hierarchia Catholica medii et recentioris aevi, sive summorum pontificum, S.R.E. cardinalium, ecclesiarum antistitum series volumen tertium saeculum XVI ab anno 1503 complectens, Monasterii (Münster), 1923.

FAlguiÈRes GUIDICELli, Patricia, La cité fictive. Les collections de cardinaux, à Rome, au XVI siècle, en André CHASTEL (ed.), Les Carrache et les Décors Profanes (Roma, 2-4 de octubre 1986), École Française de Rome, Rome, 1988, pp. 215-333.

FERNÁNDEZ DE CóRDOVA MIRALles, Álvaro, Imagen de los Reyes Católicos en la Roma pontificia, en En la España medieval, 28 (2005), pp. 259-354.

- López de Carvajal, Bernardino, en Diccionario Biográfico Español, vol. XXX, 2009, pp. 395-401.

- Diplomáticos y letrados en Roma al servicio de los Reyes Católicos: Francesco Vitale di Noya, Juan Ruiz de Medina y Francisco de Rojas, en Dicenda: Estudios de lengua y literatura españolas, 32 (2014), pp. 113-154.

FIENGA, Doris D., The Antiquarie Prospetiche Romane composte per Prospectivo Melanese Depictore: A document for the study of the relationship between Bramante and Leonardo Da Vinci, Tesis Doctoral (1970), University of California, Los Ángeles, 1971.

FRAY MARIANO DA FIRENZE, Itinerarium Urbis Romae, ed. por BulletTI O.F.M., Roma, 1931.

FreIBERG, Jack, Bramante's Tempietto, Roman Renaissance, and the Spanish Crown, Cambridge University Press, Cambridge, 2014.

Frommel, Luipolt C., Progetto e archeologia in due disegni di Antonio da Sangallo il Giovane per Santa Croce in Gerusalemme, en S. Danesi SQUARZINA (ed.), Roma, centro ideale della cultural dell'antico nei secoli XV e XVI. Da Martino V al sacco di Roma, 1417-1527, Milano, 1989, pp. 382-389.

GÓMEZ MOREnO, Ángel, España y la Italia de los humanistas: primeros ecos, Madrid, 1994.

GONZÁleZ GERMAIN, Gerard, La silloge epigrafica di Pietro Sabino: un riesame della tradizione manoscritta, en Epigraphica, 78 (2016), pp. 315-335.

GOÑ GAZTAMBIDE, José, Bernardino López de Carvajal y las bulas alejandrinas, en Anuario de Historia de la Iglesia, 1 (1992), pp. 93-112.

GIONTA, Daniela, Epigrafia umanistica a Roma, Messina, 2005.

GIUSTINIANI, Michele, Due libri de vescovi e de governatori di Tivoli, Roma, 1665.

GOVI, Gilberto, Intorno a un opusculo rarissimo della fine del secolo XV intitolato «Antiquarie Prospettiche Romane» composte per Prospettivo Milanese Dipintore, en Atti della Reale Accademia dei Lincei, 2a s., 3, 1876, pp. 49-66. 


\section{PALOMA MARTÍN-ESPERANZA}

GRAFTON, Anthony, Invention of Traditions and Traditions of Invention in Renaissance Europe: The Strange Case of Annius of Viterbo, en Anthony GRAFTON y Ann BLAIR (eds.), The Transmission of Culture in Early Modern Europe, 1990, Philadephia, pp. 8-38.

Greswell, William Parr, Angelo Poliziano, Giovanni Pico della Mirandola, Jacopo Sannazaro, Pietro Bembo, Girolamo Fracastoro, Marco Antonio Flaminio, Girolamo Amalteo, Giovanni Battista Amalteo, and Cornelio Amalteo. Memoirs of Angelus Politianus, Foannes Picus of Mirandula, Actius Sincerus Sannazarius, Petrus Bembus, Hieronymus Fracastorius, Marcus Antonius Flaminius, and the Amalthei: Translations from Their Poetical Works: and Notes and Observations Concerning Other Literary Characters of the Fifteenth and Sixteenth Centuries, Cadell \& Davies, London, 1805.

GUICCIARDINI, Francesco, Storia d'Italia, vI/2, ed. por S. SEIDEL MENCHI, Turín, 1971.

HERNANDO SÁNCHEZ, Carlos José (coord.), Roma y España. Un crisol de la cultura europea en la Edad Moderna, 2 vols., Madrid, 2007.

IANNUZZI, Isabella, Bernardino de Carvajal: teoria e propaganda di uno spagnolo all'interno della Curia romana, en Rivista di storia della Chiesa in Italia, 62/1 (2008), pp. 25-45.

- Le radici culturali di uno spagnolo alla corte papale: Bernardino de Carvajal, en Metafore di un pontificato. Giulio II (1503-1513), (Roma, 2-3 de diciembre de 2008), Roma, 2010, pp. 45 59.

INFESSURA, Stefano, Diario de la città di Roma di Stefano Infessura scribasenato, ed. O. Tommasini, Roma, 1890.

Korhonen, Kalle, Le iscrizioni del Museo Civico di Catania. Storia delle collezioni-Cultura epigrafica-Edizione (Commentationes Humanarum Litterarum, 121), Helsinki, 2004.

LANCIANI, Roberto, Storia degli scavi di Roma e notizie intorno le collezioni romani di antichità, I (1000-1530), Roma, 1989.

LigOrio, Pirro, Libro dell'antica città di Tivoli e di alcune famose ville, ed. Alessandra TEN, Roma, 2005.

MAgISTER, Sara, Censimento dell collezioni di antichità a Roma (1471-1503): addenda, en Xenia Antiqua, X (2001), pp. 113-154.

- Censimento dell collezioni di antichità a Roma: 1471-1503, en Xenia Antiqua, VIII (1999), pp. 129-204.

- Le collezione di Antichità del cardinale Giuliano della Rovere. Anteprima di studio, en Xenia antiqua, IX (2000), pp. 47-50.

MARÍAS, Fernando, Los clientes del Tempietto: historia, intenciones y significados, en Flavia CANTATORE (ed.), Il Tempietto di Bramante nel monasterio di San Pietro in Montorio, Edizioni Quasar, Roma, 2017, pp. 111-152.

MARINI, Luigi Gaetano, Degli archiatri pontifici, I, Roma, 1784, pp. 218-227.

MARTÍN-EsPeranZA, Paloma, Alejandro VI y las antigüedades de Roma: coleccionismo, excavaciones y spolia, en Revista Borja. Revista de l'IIEB, 6 (2017-2018), pp. 1-28.

MINASI, Mara, Passione politica e travestimento all'antica: la collezione antiquaria della familia Porcari, en Anna Cavallaro (ed.), Collezioni di Antichità a Roma tra '400 e '500, De Luca Editori d'Arte, Roma, 2007, pp. 83-104.

MORA, Gloria, La escultura clásica y los estudios sobre la Antigüedad en España en el siglo XVI: colecciones, tratados y libros de diseños, en Matteo MANCINI (coord.), El coleccionismo de escultura clásica en España: actas del simposio: 21 y 22 de mayo de 2001, Madrid, 2001, pp. 115-142. 


\section{POLÍTICA Y MECENAZGO ANTICUARIO EN LA ROMA DEL RENACIMIENTO}

MÜNTZ, Eugène, Les antiquités de la ville de Roma aux XIV, XV et XVI siècles, Ernest Leroux, París, 1886.

- Les arts à la cour des Papes pendant le XV et le XVI siècles, III: Sixte IV - Léon X (1471-1521), París: Ernest Leroux, 1882.

- Les arts à la cour des Papes pendant le XV et le XVI siècles, IV, París, Ernest Leroux, 1898.

PaOlinI, Devid, Los Reyes Católicos e Italia: los humanistas italianos y su relación con España, en Nicasio Salvador Miguel y Cristina Moya García (eds.), La literatura en la época de los Reyes Católicos, Pamplona-Madrid-Frankfurt, 2008, pp. 189-205.

PAOLUZZI, Maria Cristina, La familia della Valle e l'origine della collezione di antichità, en Anna Cavallaro (ed.), Collezioni di Antichità a Roma tra '400 e '500, De Luca Editori d'Arte, Roma, 2007, pp. 147-186.

Pereda, Felipe, Pedro González de Mendoza, de Toledo a Roma. El patronazgo de Santa Croce in Gerusalemme: entre la arqueología y la filología, en Frédérique LEMERLE, Yves PAUWELS, Gennaro Toscano (eds.), Les Cardinaux de la Renaissance et la modernité artistique, Publications de l'Institut de recherches historiques du Septentrion, Villeneuve d'Ascq, 2012, pp. 217 243.

Piccolomini, Enea Silvio, I Commentarii, ed. Giuseppe Bernetti, 5 vol., Cantagalli, Siena, 1972-1976.

Salvador Miguel, Nicasio, Intelectuales españoles en Roma durante el gobierno de los Reyes Católicos, en Patrizia BOTTA (coord.), Rumbos del humanismo en el umbral del cincuentenario de la A[sociación] I[nternacional de] H[ispanistas], Roma, 2012, pp. 47-64.

SaNTOLINI, Sandro, Pietro e Mario Millini fondatori di una dinastía di collezionisti antiquari, en Anna Cavallaro (ed.), Collezioni di Antichità a Roma tra '400 e '500, De Luca Editori d'Arte, Roma, 2007, pp. 39-62.

- Pietro e Mario Millini fondatori di una dinastía di collezionisti antiquari, en Anna Cavallaro (ed.), Collezioni di Antichità a Roma tra '400 e '500, De Luca Editori d'Arte, Roma, 2007, pp. 39-62.

SCHMITT, Anne, Römische Antikensammlungen im Spiegel eines Musterbuch der Renaissance, en Müncher Fabrbuch der bildenden Kunst, 21 (1970), pp. 99-128.

SODIN, Heikki, Appunti su Battista Brunelleschi epigrafista, en O. MERISALO y R. VAINIO (eds.), Ad itum liberum. Essays in honour of Anne Helttula, Jyväskylä, 2007, pp. 79-92.

SPERINDEI, Simona, Repertorio delle residenze cardenalizie, en Giorgio Simoncini (ed.), Roma. Le trasformazioni urbane nel Quattrocento. II. Funzione urbane e tipologie edilizie (L'Ambiente Storico, en Studi di Storia urbana e del territorio, XI), Firenze, 2004, pp. 137-158.

STEPHENs, Walter, When Pope Noab Ruled the Etruscans: Annius of Viterbo and his Forged Antiquities, en MLN (Mod Lang Notes) 119/1 (2004), pp. 201-223.

VICARELli, Francesca, La collezione di antichità della familia Santacroce, en Anna CAVALLARO (ed.), Collezioni di Antichità a Roma tra '400 e '500, De Luca Editori d'Arte, Roma, 2007, pp. 63-82. 
0000 